\title{
Atezolizumab in locally advanced or metastatic urothelial cancer: a pooled analysis from the Spanish patients of the IMvigor 210 cohort 2 and 211 studies
}

\author{
M. Sotelo ${ }^{1} \cdot$ T. Alonso-Gordoa ${ }^{2}$ P. Gajate ${ }^{2} \cdot$ E. Gallardo ${ }^{3} \cdot$ R. Morales-Barrera ${ }^{4}$ J. L. Pérez-Gracia ${ }^{5} \cdot$ J. Puente $^{6}$. \\ P. Sánchez ${ }^{7} \cdot$ D. Castellano ${ }^{8} \cdot$ I. Durán $^{1,9}$ (D)
}

Received: 5 June 2020 / Accepted: 18 August 2020 / Published online: 8 September 2020

(c) The Author(s) 2020

\begin{abstract}
Background The studies IMvigor 210 cohort 2 and IMvigor211 evaluated the efficacy of atezolizumab in patients with locally advanced or metastatic urothelial cancer (mUC) upon progression to platinum-based chemotherapy worldwide. Yet, the real impact of this drug in specific geographical regions is unknown.

Materials and methods We combined individual-level data from the 131 patients recruited in Spain from IMvigor 210 cohort 2 and IMvigor211 in a pooled analysis. Efficacy and safety outcomes were assessed in the overall study population and according to PD-L1 expression on tumour-infiltrating immune cells.

Results Full data were available for 127 patients; 74 (58\%) received atezolizumab and 53 (42\%) chemotherapy. Atezolizumab patients had a numerically superior median overall survival although not reaching statistical significance $(9.2$ months vs 7.7 months). No statistically significant differences between arms were observed in overall response rates ( $20.3 \%$ vs $37.0 \%)$ or progression-free survival (2.1 months vs 5.3 months). Nonetheless, median duration of response was superior for the immunotherapy arm (non-reached vs 6.4 months; $p=0.005$ ). Additionally, among the responders, the 12-month survival rates seemed to favour atezolizumab $(66.7 \%$ vs $19.9 \%)$. When efficacy was analyzed based on PD-L1 expression status, no significant differences were found. Treatment-related adverse events of any grade occurred more frequently in the chemotherapy arm [46/57 (81\%) vs 44/74 (59\%)].

Conclusion Patients who achieved an objective response on atezolizumab presented a longer median duration of response and numerically superior 12 month survival rates when compared with chemotherapy responders along with a more favorable safety profile. PD-L1 expression did not discriminate patients who might benefit from atezolizumab.
\end{abstract}

Keywords Atezolizumab $\cdot$ Metastatic urothelial cancer $\cdot$ Duration of response $\cdot$ Spain

I. Durán

ignacioduranmartinez@gmail.com

1 Marqués de Valdecilla University Hospital, Edificio Sur. Despacho 277, Avda Valdecilla s/n, 39005 Santander, Spain

2 Ramon y Cajal University Hospital, Madrid, Spain

3 Parc Taulí Hospital Universitari, Institut d'Investigació i Innovació Parc Taulí I3PT, Universitat Autònoma de Barcelona, Sabadell, Spain

4 Vall d'Hebron University Hospital, Barcelona, Spain
5 University Clinic of Navarra, Pamplona, Spain

6 Medical Oncology Department, Hospital Clínico San Carlos, Instituto de Investigación Sanitaria del Hospital Clínico San Carlos (IdISSC), CIBERONC, Madrid, Spain

7 Medical Department, Roche Farma S.A., Madrid, Spain

8 Doce de Octubre University Hospital, Madrid, Spain

9 Instituto de Investigación Marqués de Valdecilla (IDIVAL), Santander, Spain 


\section{Introduction}

Urothelial cancer (UC) is a frequent disease globally with over 549,000 new diagnoses worldwide in 2018 [1]. The incidence and mortality of UC differ across countries due to differences in risk factors, diagnostic practices and accessibility of therapies. In Spain, the incidence of this tumour is particularly high, ranking fifth altogether and rising up to the fourth most frequent malignancy among males. In 2020, more than 22,000 diagnoses of UC are expected in our country representing a relevant medical challenge [2]. Around 70\% of UC diagnoses are in early stages (non-muscle-invasive disease) where prognosis after appropriate treatment remains overall favourable with a 5 year overall survival (OS) exceeding $90 \%$. Nevertheless, $25 \%$ of patients present with disease invading the Muscularis propria or beyond (muscle-invasive UC) and despite optimal management, roughly $40-50 \%$ will relapse, presenting metastatic disease not amenable for surgical treatment. Additionally, about 5\% of the new diagnoses present with advanced disease at diagnosis. Metastatic UC harbours a poor prognosis with a 5 year OS that historically does not exceed 10-15\% [3, 4]. Chemotherapy regimens based on cisplatin have become the most utilized ones in this setting. The cisplatin-gemcitabine (CG) combination provides objective response rates (ORR) in the range of $50 \%$ with $12 \%$ of complete responses (CR) and a median OS of around 15 months [5]. Despite these promising results, two facts have historically limited progress in advanced UC therapeutics. First, about $50 \%$ of patients with metastatic disease are considered unfit to receive cisplatin according to predefined criteria (the Galsky's criteria). These patients can only receive alternative regimens, such as carboplatin, or other regimes with inferior outcomes to cisplatin $[6,7]$. Second, nearly all patients with metastatic disease, regardless of their response to first-line treatment, will end up progressing and the classic chemotherapy agents explored in the second line have historically provided scarce benefit with an ORR of less than $10 \%$, a short duration of response (DoR) and a median OS of about 7 months [8]. Therefore, defining a better treatment for cisplatin-unfit patients and improving poor outcomes in the second-line setting are unmet needs.

In this context, a different approach to treat $\mathrm{UC}$ has been developed considering specific features of this tumour [9]. One distinctive characteristic of UC is the high rate of somatic mutations observed that may potentially increase the capacity of the host immune system to distinguish UC cells as foreign. This would eventually lead to proper tumour cell identification and elimination [10]. Nevertheless, UC cells might escape immune surveillance through the expression of programmed death-ligand
1 (PD-L1) in the tumour microenvironment [11-13]. Back in 2014, an expansion cohort of about seventy patients with heavily pretreated advanced UC was treated with an anti-PD-L1 antibody (i.e. atezolizumab) as part of a multitumour phase I study. Beyond positive safety data, unprecedented activity was reported with ORR in the range of $40-50 \%$ in patients whose tumours highly expressed PD-L1-positive tumour-infiltrating immune cells [14]. These results led to considering testing this compound in advanced UC patients beyond platinum progression and, due to the favourable toxicity profile, this drug appeared as an option for cisplatin-unfit patients. Hence, two separate trials (IMvigor 210 and IMvigor 211) were launched and tested atezolizumab in different populations. IMvigor 210 was a phase 2 study with two single-arm cohorts where patients either treatment naïve unfit for cisplatin (cohort 1) or with progression beyond first-line chemotherapy (cohort 2) received atezolizumab with the ORR as the primary endpoint. The positive results of these two cohorts moved atezolizumab development forward and led to drug approval and the incorporation of this compound in treatment algorithms [15, 16]. Across drug development, the expression of PD-L1 appeared as a potential positive predictor of response to immunotherapy. Atezolizumab was subsequently tested in a randomized phase 3 study (IMvigor 211) that compared chemotherapy vs. atezolizumab in patients with advanced UC and progression beyond platinum-based chemotherapy. The primary endpoint of OS was tested hierarchically in pre-specified populations according to the PD-L1 expression assuming greater benefit to atezolizumab in patients with higher PD-L1 expression. Nevertheless, there were no differences regarding OS according to this design and PD-L1 expression behaved as a prognostic rather than predictive factor, favouring also the chemotherapy arm. Yet, atezolizumab showed longer DoR, was better tolerated and was superior to chemotherapy in landmark analyses $[17,18]$.

We report data from an individual-level pooled analysis of the subset of Spanish patients in the IMvigor 210 cohort 2 and IMvigor211 studies to explore whether their outcomes differed from overall results or not.

\section{Materials and methods}

We analyzed the efficacy and safety of atezolizumab in Spanish patients that were recruited in the cohort 2 of the IMvigor 210 and IMvigor 211 studies. Patients of both studies were aged $\geq 18$ years with advanced or metastatic urothelial carcinoma whose disease had progressed after previous platinum-based chemotherapy Baseline characteristics are presented in Table 1. 
Table 1 Baseline characteristics

ITT popula- IC0/1 popula- IC2/3 popula- Atezolizumab $(n=74) \quad$ Chemotherapy $(n=57)$ tion $(n=131) \quad$ tion $(n=98) \quad$ tion $(n=33)$

\begin{tabular}{|c|c|c|c|c|c|}
\hline \multicolumn{6}{|l|}{ Age } \\
\hline Median (years; range) & $66(41-85)$ & $67.5(41-85)$ & $64.0(4-83)$ & $68.0(41-85)$ & $66.0(47-84)$ \\
\hline$\geq 80$ years & $8(6.1 \%)$ & $6(6.1 \%)$ & $2(6.1 \%)$ & $7(9.5 \%)$ & $1(1.8 \%)$ \\
\hline \multicolumn{6}{|l|}{ Sex } \\
\hline Female & $23(17.6 \%)$ & $18(18.4 \%)$ & $5(15.2 \%)$ & $13(17.6 \%)$ & $10(17.5 \%)$ \\
\hline Male & $108(82.4 \%)$ & $80(81.6 \%)$ & $28(84.8 \%)$ & $61(82.4 \%)$ & $47(82.5 \%)$ \\
\hline \multicolumn{6}{|l|}{ Tobacco use } \\
\hline Current & $21(16.0 \%)$ & $17(17.3 \%)$ & $4(12.1 \%)$ & $14(18.9 \%)$ & $7(12.3 \%)$ \\
\hline Former & $26(19.8 \%)$ & $20(20.4 \%)$ & $6(18.2 \%)$ & $20(27.0 \%)$ & $6(10.5 \%)$ \\
\hline Never & $84(64.1 \%)$ & $61(62.2 \%)$ & $23(69.7 \%)$ & $40(54.1 \%)$ & $44(77.2 \%)$ \\
\hline \multicolumn{6}{|l|}{ Primary tumour site } \\
\hline Renal pelvis & $12(9.2 \%)$ & $9(9.2 \%)$ & $3(9.1 \%)$ & $4(5.4 \%)$ & $8(14.0 \%)$ \\
\hline Ureter & $17(13.0 \%)$ & $12(12.2 \%)$ & $5(15.2 \%)$ & $12(16.2 \%)$ & $5(8.8 \%)$ \\
\hline Bladder & $100(76.3 \%)$ & $75(76.5 \%)$ & $25(75.8 \%)$ & $56(75.7 \%)$ & $44(77.2 \%)$ \\
\hline Urethra/Other & $2(1.5 \%)$ & $2(2.0 \%)$ & $0(0.0 \%)$ & $2(2.7 \%)$ & 0 \\
\hline Metastatic disease & $108(82.4 \%)$ & $82(83.7 \%)$ & $26(78.8 \%)$ & $64(86.5 \%)$ & $44(77.2 \%)$ \\
\hline \multicolumn{6}{|l|}{ Site of metastases } \\
\hline Visceral & $96(73.3 \%)$ & $73(74.5 \%)$ & $23(69.7 \%)$ & $59(79.7 \%)$ & $37(64.9 \%)$ \\
\hline Liver & $32(24.4 \%)$ & $24(24.5 \%)$ & $8(24.2 \%)$ & $19(25.7 \%)$ & $13(22.8 \%)$ \\
\hline \multicolumn{6}{|l|}{ ECOG performance status } \\
\hline 0 & $53(40.5 \%)$ & $40(40.8 \%)$ & $13(39.4 \%)$ & $34(45.9 \%)$ & $19(33.3 \%)$ \\
\hline 1 & $78(59.5 \%)$ & $58(59.2 \%)$ & $20(60.6 \%)$ & $40(54.1 \%)$ & $38(66.6 \%)$ \\
\hline Serum haemoglobin $<10 \mathrm{~g} / \mathrm{dl}$ & $14(10.7 \%)$ & $13(13.3 \%)$ & $1(3.0 \%)$ & $8(10.8 \%)$ & $6(10.5 \%)$ \\
\hline \multicolumn{6}{|l|}{ Number of risk factors } \\
\hline 0 & $40(30.5 \%)$ & $30(30.6 \%)$ & $10(30.3 \%)$ & $25(33.8 \%)$ & $15(26.3 \%)$ \\
\hline 1 & $64(48.9 \%)$ & $46(46.9 \%)$ & $18(54.5 \%)$ & $34(45.9 \%)$ & $30(52.6 \%)$ \\
\hline 2 & $21(16.0 \%)$ & $17(17.3 \%)$ & $4(12.1 \%)$ & $12(16.2 \%)$ & $9(15.8 \%)$ \\
\hline 3 & $6(4.6 \%)$ & $5(5.1 \%)$ & $1(3.0 \%)$ & $3(4.1 \%)$ & $3(5.3)$ \\
\hline Previous cystectomy & $62(47.3 \%)$ & $45(45.9 \%)$ & $17(51.5 \%)$ & $30(40.5 \%)$ & $32(56.1 \%)$ \\
\hline Time from previous chemotherapy $<3$ months & $43(32.8 \%)$ & $35(35.7 \%)$ & $8(24.2 \%)$ & $26(35.1 \%)$ & $17(29.8 \%)$ \\
\hline \multicolumn{6}{|c|}{ Number of previous systemic regimens in the metastatic setting } \\
\hline 0 & $43(35.1 \%)$ & $29(29.6 \%)$ & $14(42.4 \%)$ & $20(27.0 \%)$ & $23(40.4 \%)$ \\
\hline 11 & $59(45.0 \%)$ & $48(49.0 \%)$ & $11(33.3 \%)$ & $34(45.9 \%)$ & $25(43.9 \%)$ \\
\hline 2 & $23(17.6 \%)$ & $17(17.3 \%)$ & $6(18.2 \%)$ & $14(18.9 \%)$ & $15(26.3 \%)$ \\
\hline$\geq 3$ & $6(4.6 \%)$ & $4(4.1 \%)$ & $2(6.1 \%)$ & $6(8.1 \%)$ & 0 \\
\hline \multicolumn{6}{|l|}{ Previous systemic regimen setting } \\
\hline Metastatic & $88(67.2 \%)$ & $69(70.4 \%)$ & $19(57.6 \%)$ & $54(73.0 \%)$ & $34(59.6 \%)$ \\
\hline $\begin{array}{l}\text { Neoadjuvant or adjuvant chemotherapy with } \\
\text { progression within } \leq 12 \text { months }\end{array}$ & $42(32.1 \%)$ & $28(28.6 \%)$ & $14(44.4 \%)$ & $19(25.7 \%)$ & $23(40.4 \%)$ \\
\hline Other & $1(0.8 \%)$ & $1(1.0 \%)$ & 0 & $1(1.4 \%)$ & 0 \\
\hline
\end{tabular}

Data are median (range), $n(\%)$, or $n / N(\%)$, unless otherwise specified

Once data from both studies were combined, we described the ORR, DoR, PFS and OS, of patients who received atezolizumab (from both IMvigor210 and 211 studies) or chemotherapy (from IMvigor211), in the modified ITT population (all the subjects with their response assessed) and according to the PD-L1 expression (Ventana PD-L1 SP142 assay) on tumour-infiltrating immune cells (IC0/1 if $<5 \%$ of tumour-infiltrating immune 
expressed PD-L1 or IC2/3 if $\geq 5 \%$ expressed PD-L1). Safety outcomes were described in the ITT (all the randomized subjects).

Patients received atezolizumab $1200 \mathrm{mg}$ or chemotherapy (vinflunine $320 \mathrm{mg} / \mathrm{m}^{2}$, paclitaxel $175 \mathrm{mg} / \mathrm{m}^{2}$ or docetaxel $75 \mathrm{mg} / \mathrm{m}^{2}$ based on the physician's choice), intravenously every 3 weeks until unacceptable toxicity, RECIST v1.1 progression, or informed consent withdrawal.

\section{Results}

Our sample comprised $21(6.6 \%)$ patients out of the 315 recruited in the cohort 2 of the IMvigor 210 study plus $110(11.8 \%)$ out of the 931 subjects in the IMvigor 211 study. These 131 patients treated in Spanish centers were assigned to either atezolizumab $(n=74 ; 56.5 \%)$ or chemotherapy $(n=57 ; 43.5 \%)$ and made up the intention-to-treat (ITT) population. However, the response was missing in 4 patients, and therefore the resulting 127 patients made up the modified ITT (mITT) population for efficacy outcomes, 74 $(58.3 \%)$ on atezolizumab and $53(41.7 \%)$ on chemotherapy (67.9\% vinflunine and $32.1 \%$ taxanes).

Analysis of the efficacy data revealed no statistically significant differences across arms in ORR with 12 (20.3\%) responses out of the 59 evaluable patients in the atezolizumab arm compared with 17 (37.0\%) out of the 46 patients in the chemotherapy arm $(p=0.059)$. Nonetheless, in those patients who responded, the DoR clearly favored the atezolizumab arm. Median DoR was not reached for atezolizumab vs 6.4 months for chemotherapy being these differences statistically significant (HR 0.24, 95\% CI 0.07-0.66; $p=0.005$ ) (Fig. 1). Moreover, remarkable differences were observed in indicators of long-term benefit. Among those patients who presented either a partial or complete response (12 and 17 patients in atezolizumab and chemotherapy, respectively), the 6-month survival rates were quite different in favor of the immunotherapy group. Thus, $91.7 \%$ of atezolizumab treated responders were alive at 6 months versus $64.5 \%$ of patients who received chemotherapy. This trend was maintained overtime and when analyzing the long-term efficacy, the differences between the two treatment arms were even larger. Hence, the 12 month survival rates were $66.7 \%$ and $19.9 \%$ for immunotherapy and chemotherapy patients, respectively.

Although the median progression-free survival (PFS) was 5.3 months (95\% CI 3.7-6.7) in the chemotherapy arm compared to 2.1 months (95\% CI 2.0-2.5) in the atezolizumab arm $(\mathrm{p}=0.043), 12$-month PFS rates were not significantly different ( $p=0.0574), 12.2 \%$ (95\% CI 5.0-22.8) for chemotherapy and 9.1\% (95\% CI 3.4-18.4) for atezolizumab.

Median OS was numerically superior in the atezolizumab group compared to the chemotherapy group, (9.2 months; 95\% CI 6.5-11.7 vs 7.7 months; 95\% CI 5.3-10.8) albeit not reaching statistical significance (Fig. 2). However, patients in the atezolizumab group did have fewer events than in the chemotherapy group, $(66.2 \%$ vs $84.9 \% ; p=0.018)$ Table 2 .
Fig. 1 Duration of response in the mITT population. HR 0.24 , 95\% CI $0.07-0.66 ; p=0.005$

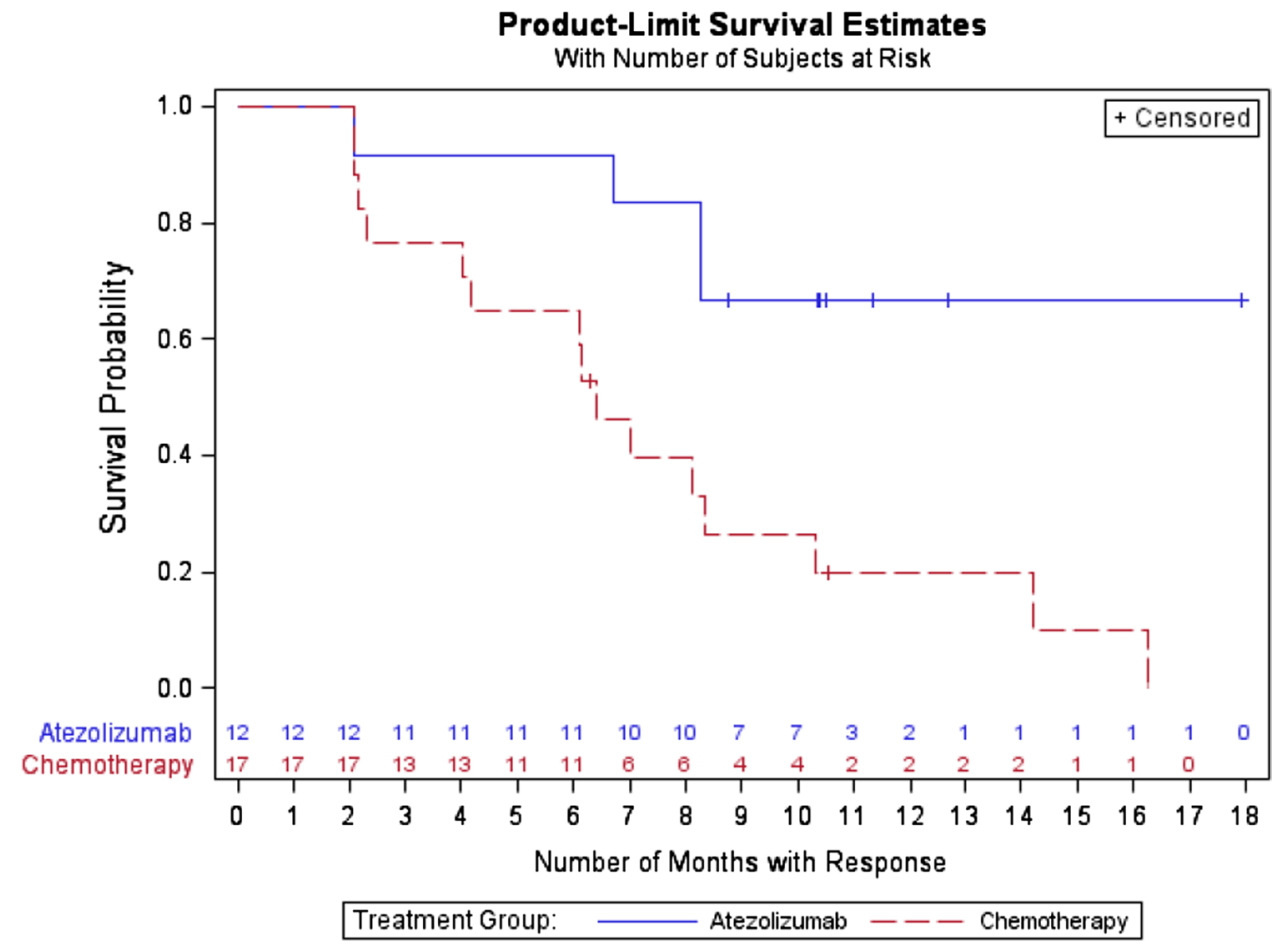


Fig. 2 Overall survival in the mITT population. HR $0.86,95 \%$ CI $0.57-1.29 ; p=0.462$

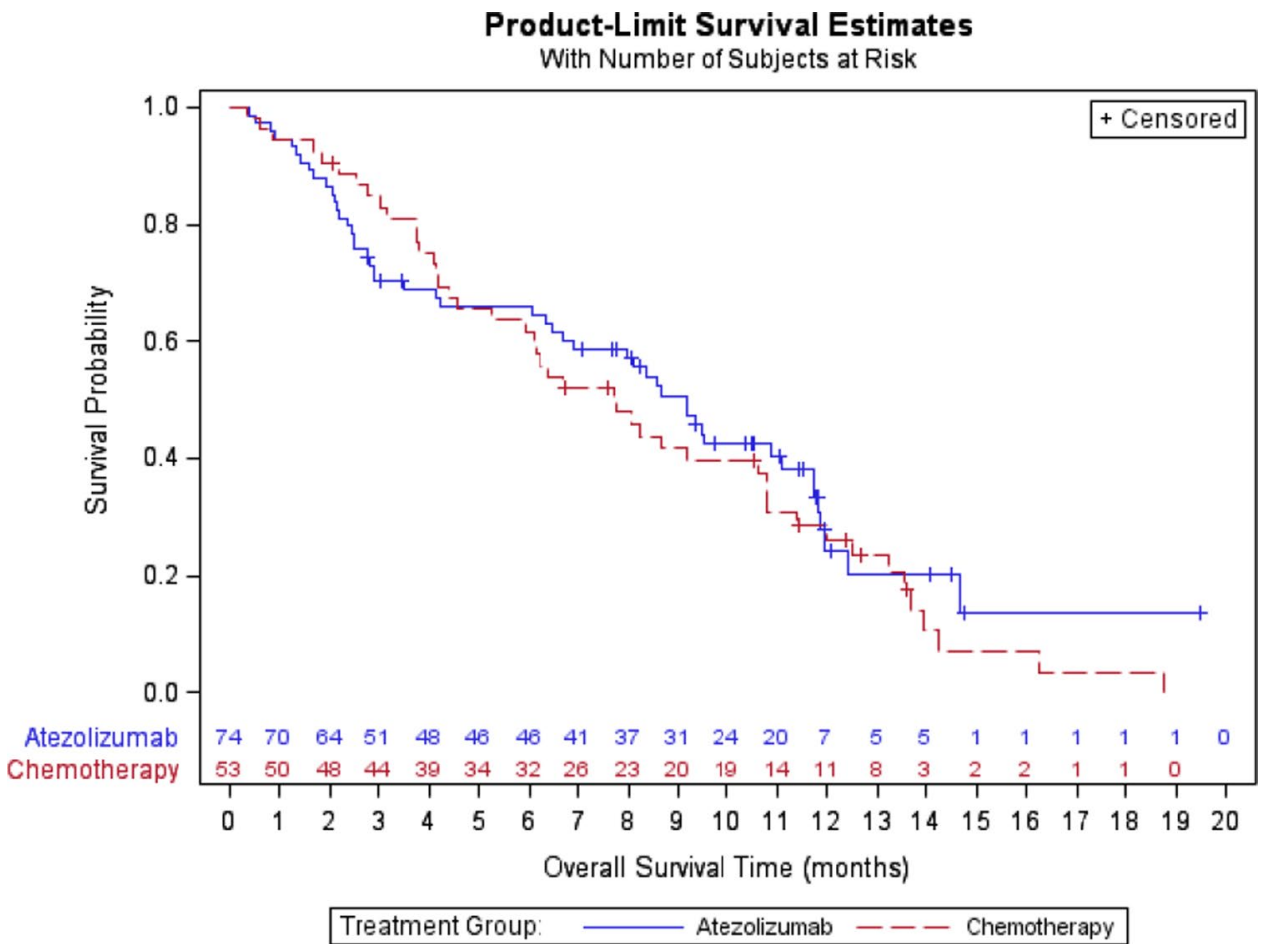

Interestingly, when the efficacy analysis was performed stratifying patients according to the PD-L1 expression status on infiltrating immune cells (ICs) in the tumour microenvironment, our analyses did not find any significant difference either in ORR, DoR, PFS or OS regardless of the IC group in patients treated with either atezolizumab or chemotherapy (Table 2).

The safety analysis revealed that any-grade treatmentrelated adverse events occurred in 44/74 (59\%) atezolizumab patients vs. $46 / 57$ (81\%) chemotherapy patients. Grade 3-4 adverse events were documented in $6(8 \%)$ patients given atezolizumab vs. 28 (49\%) given chemotherapy (Fig. 3). Among patients on chemotherapy, grade 3-4 AEs occurred in $57.5 \%$ of patients allocated to vinflunine and in $29 \%$ of patients assigned to taxanes.

The most frequent adverse events (those who affected to at least $10 \%$ of patients) were asthenia, pruritus and diarrhoea in patients treated with atezolizumab. For patients on chemotherapy, the commonest adverse events were asthenia, neutropenia, constipation, alopecia, abdominal pain, anaemia, nausea, diarrhoea, mucositis, decreased appetite and vomiting. The most common grade 3-4 adverse events in the chemotherapy group were neutropenia (28\%), asthenia (12\%), constipation (9\%), anaemia (7\%) and abdominal pain (5\%). A detailed list of treatment-related AEis by frequency and severity is presented in Table 3 .

\section{Discussion}

For more than three decades, no progress has been made in advanced UC therapeutics [8]. Yet, the recent incorporation of immunotherapy with the arrival to the clinic of the check-point inhibitors has reshaped the treatment scenario, and both atezolizumab (an anti-PD-L1) and pembrolizumab (an anti-PD-1) have become standard options for patients with advanced UC with either progression beyond first-line chemotherapy or treatment naïve and unfit to receive cisplatin [19]. Immunotherapy has demonstrated substantial benefit in this patient population and a better toxicity profile across different trials [15, 16, 20, 21]. Nevertheless, there are still unanswered questions about the real impact and whether clinical or biological factors might help us predict the efficacy of this innovative treatment strategy or not [22]. Sub-analyses of certain populations in this context could unveil details relevant for a better understanding of the activity and safety and could help explain unexpected results or even pose new questions that might deserve to be answered. A pooled analysis of the Spanish patients in two relevant trials of atezolizumab in advanced UC patients is presented here. When analyzing efficacy, ORR showed no statistically significant differences between the immunotherapy and the chemotherapy arms as previously reported in other atezolizumab studies, such as IMvigor 211, where ORR was 
Table 2 Efficacy outcomes

\begin{tabular}{|c|c|c|c|c|c|c|}
\hline \multirow[t]{2}{*}{ PD-L1 score } & \multicolumn{2}{|l|}{ mITT } & \multicolumn{2}{|l|}{$\mathrm{IC} 0 / 1$} & \multicolumn{2}{|l|}{$\mathrm{IC} 2 / 3$} \\
\hline & $\begin{array}{l}\text { Atezolizumab } \\
(n=74)\end{array}$ & $\begin{array}{l}\text { Chemotherapy } \\
(n=53)\end{array}$ & $\begin{array}{l}\text { Atezolizumab } \\
(n=54)\end{array}$ & $\begin{array}{l}\text { Chemotherapy } \\
(n=41)\end{array}$ & $\begin{array}{l}\text { Atezolizumab } \\
(n=20)\end{array}$ & $\begin{array}{l}\text { Chemotherapy } \\
(n=12)\end{array}$ \\
\hline \multicolumn{7}{|l|}{ Overall survival $^{\mathrm{a}}$} \\
\hline $\begin{array}{l}\text { Patients with } \\
\text { event }(\%)\end{array}$ & $49(66.2 \%)$ & $45(84.9 \%)$ & $37(68.5 \%)$ & $36(87.8 \%)$ & $12(60.0 \%)$ & $9(75.0 \%)$ \\
\hline $\begin{array}{l}\text { Median (months; } \\
\text { 95\% CI) }\end{array}$ & $9.2(6.5-11.7)$ & $7.7(5.3-10.8)$ & $9.2(6.5-11.7)$ & $6.4(4.1-8.6)$ & $10.9(2.4-12.4)$ & $12.0(4.2-13.6)$ \\
\hline $\begin{array}{l}6 \text { month overall } \\
\text { survival rate } \\
(95 \% \mathrm{CI})\end{array}$ & $65.9 \%(53.8-75.5)$ & $61.7 \%(47.1-73.3)$ & $68.4 \%(54.2-79.0)$ & $56.1 \%(39.7-69.6)$ & $59.2 \%(34.7-77.2)$ & $81.8 \%(44.7-95.1)$ \\
\hline $\begin{array}{l}12 \text { month overall } \\
\text { survival rate } \\
(95 \% \mathrm{CI})\end{array}$ & $24.4 \%(13.1-37.6)$ & $26.2 \%(14.8-39.1)$ & $22.3 \%(10.5-36.9)$ & $21.8 \%(10.4-35.9)$ & $37.6 \%(15.2-60.1)$ & $41.6 \%(13.1-68.4)$ \\
\hline \multicolumn{7}{|c|}{ Progression-free survival ${ }^{\mathrm{b}}$} \\
\hline $\begin{array}{l}\text { Patients with } \\
\text { event (\%) }\end{array}$ & $67(90.5 \%)$ & $49(92.5 \%)$ & $51(94.4 \%)$ & $39(95.1 \%)$ & $16(80.0 \%)$ & $10(83.3 \%)$ \\
\hline $\begin{array}{l}\text { Median (months; } \\
\text { 95\% CI) }\end{array}$ & $2.1(2.0-2.5)$ & $5.3(3.7-6.7)$ & $2.1(2.0-2.5)$ & $4.2(2.2-7.7)$ & $2.2(1.8-4.2)$ & $5.3(3.4-10.4)$ \\
\hline $\begin{array}{l}12 \text { month PFS } \\
\text { rate }(95 \% \mathrm{CI})\end{array}$ & $9.1 \%(3.4-18.4)$ & $12.2 \%(5.0-22.8)$ & $6.9 \%(2.0-16.1)$ & $10.4 \%(3.3-22.1)$ & $20.0 \%(6.2-39.3)$ & $18.2 \%(2.9-44.2)$ \\
\hline \multicolumn{7}{|c|}{ Objective response rate } \\
\hline $\begin{array}{l}\text { No. of evaluable } \\
\text { patients }\end{array}$ & 59 & 46 & 43 & 34 & 16 & 12 \\
\hline $\begin{array}{l}\text { No. of patients } \\
\text { with response } \\
(\% ; 95 \% \mathrm{CI})\end{array}$ & $\begin{array}{l}12(20.3 \% \\
11.0-32.8)\end{array}$ & $\begin{array}{l}17(37.0 \% \\
23.2-53.5)\end{array}$ & $\begin{array}{l}7(16.3 \% \\
\quad 6.8-30.7)\end{array}$ & $\begin{array}{c}12(35.3 \% \\
19.7-53.5)\end{array}$ & $\begin{array}{l}5(31.3 \% \\
\quad 11.0-58.7)\end{array}$ & $\begin{array}{l}5(41.7 \% ; \\
\quad 15.2-72.3)\end{array}$ \\
\hline \multicolumn{7}{|l|}{ Best overall response } \\
\hline $\begin{array}{l}\text { Complete } \\
\text { response }\end{array}$ & $3(4.1 \%)$ & $3(5.7 \%)$ & $1(1.9 \%)$ & $2(4.9 \%)$ & $2(10.0 \%)$ & $1(8.3 \%)$ \\
\hline Partial response & $9(12.2 \%)$ & $14(26.4 \%)$ & $6(11.1 \%)$ & $10(24.4 \%)$ & $3(15.0 \%)$ & $4(33.3 \%)$ \\
\hline Stable disease & $14(18.9 \%)$ & $18(34.0 \%)$ & $11(20.4 \%)$ & $11(26.8 \%)$ & $3(15.0 \%)$ & $7(58.3 \%)$ \\
\hline $\begin{array}{l}\text { Progressive } \\
\text { disease }\end{array}$ & $33(44.6 \%)$ & $11(20.8 \%)$ & $25(46.3 \%)$ & $11(26.8 \%)$ & $8(40.0 \%)$ & 0 \\
\hline $\begin{array}{l}\text { Missing or } \\
\text { unevaluable }\end{array}$ & $15(20.3 \%)$ & $7(13.2 \%)$ & $11(20.4 \%)$ & $7(17.1 \%)$ & $4(20.0 \%)$ & 0 \\
\hline \multicolumn{7}{|l|}{ Duration of response $\mathrm{e}^{\mathrm{c}}$} \\
\hline $\begin{array}{l}\text { Patients with } \\
\text { event }(\%)\end{array}$ & $4(33 \%)$ & $15(88 \%)$ & $3(42.9 \%)$ & $11(91.7 \%)$ & $1(20.0 \%)$ & $4(80.0 \%)$ \\
\hline $\begin{array}{l}\text { Median (months; } \\
\text { 95\% CI) }\end{array}$ & NE (6.7-NE) & $6.4(2.3-8.3)$ & NE (6.7-NE) & $6.3(2.2-10.3)$ & NA (2.1-NE) & $7.0(2.1-\mathrm{NE})$ \\
\hline $\begin{array}{l}6 \text { month survival } \\
\text { rate }(\% ; 95 \% \\
\text { CI) }\end{array}$ & $91.7 \%$ (53.9-98.8) & $64.7 \%(37.7-82.3)$ & $100 \%$ (NE-NE) & $66.7 \%(33.7-86.0)$ & $80.0 \%(20.4-96.9)$ & $60.0 \%(12.6-88.2)$ \\
\hline $\begin{array}{l}12 \text { month sur- } \\
\text { vival rate (\%; } \\
95 \% \mathrm{CI})\end{array}$ & $66.7 \%(33.7-86.0)$ & $19.9 \%(3.3-34.3)$ & $57.1 \%(17.2-83.5)$ & $20.0 \%(3.3-46.9)$ & $80.0 \%(20.4-96.9)$ & $20 \%(0.8-58.2)$ \\
\hline
\end{tabular}

${ }^{\mathrm{a} D e a t h}$

${ }^{\mathrm{b}}$ Progressive disease or death

${ }^{\mathrm{c}}$ Response stop or death 


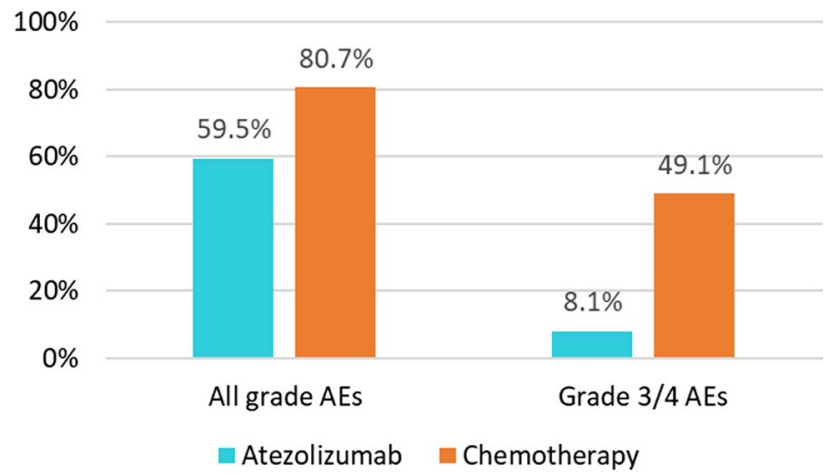

Fig. 3 Safety in the ITT population. Proportion of patients with adverse events. Data are percentages of patients presenting with AEs

almost identical in both study arms [16]. Outcomes were slightly different with pembrolizumab when compared with chemotherapy in the Keynote- 045 study where it achieved superior ORR $(21.1 \%$ vs $11.4 \% ; p=0.001)$. Whether these results could be related to less use of vinflunine over taxanes in the Keynote-045 trial remains unanswered [21]. Another parameter of efficacy, such as OS, showed also no statistically significant differences between treatment groups in our analysis. Yet, the reported numerical values are consistent with the recently reported from real-world data studies of atezolizumab and the pivotal study of pembrolizumab [21, 23].

Furthermore, regarding the analysis of the predictive value of PD-L1 staining, no significant differences in OS were observed when patients were stratified according to this biomarker. A trend towards PD-L1 expression as a favourable prognostic factor was observed. Hence, those patients IC $2 / 3$ presented a better survival in both groups and actually, OS was superior in this subgroup for the chemotherapy arm although not reaching statistical significance. This adds to other reports that suggest that PD-L1 staining is a prognostic factor rather than predictive and could justify the lack of substantial differences in efficacy in these patient's populations. Regarding PFS, the results obtained in this pooled analysis in favour of chemotherapy are overall consistent with most previous trials comparing cytotoxics with immunotherapy in different tumour types although PFS value as a surrogate marker of activity is questioned in this context [24]. Probably the most striking results of this work have to do with the long-term benefit observed in the responders in the immunotherapy group. For decades, one of the major limitations that has jeopardized progress of UC therapeutics has been the short duration of the responses presented to cytotoxic treatment. Despite relatively high ORR to chemotherapy in first line, virtually all responders will eventually relapse (most of the times early) and historical treatment options provided little or no benefit in this setting [25]. The results of our analysis illustrate remarkable differences in survival in the responders according to their treatment arm. Those patients who responded to atezolizumab presented a survival benefit at the 6 and 12 month landmarks. In the latter, around three times, more patients were alive in the atezolizumab responders compared to those who responded to chemotherapy. This behaviour appears common across different checkpoint inhibitors and different tumour types and is becoming one of the hallmarks of immunotherapy [26].

Lastly, our analysis comes to confirm the benefit of atezolizumab in terms of safety when compared with cytotoxics. As we already saw in IMvigor 211, the adverse-event profile of atezolizumab was also favourable compared with chemotherapy in the Spanish population.

The proportion of patients with any AE was lower in the atezolizumab treated patients as well as the severity of these events when compared with those who received chemotherapy. This confirms a distinctive common characteristic to most immunotherapy studies, the benefit of this treatment strategy in terms of safety, which results are particularly relevant in a patient population that normally present comorbidities. Despite the valuable information provided by these analyses, some limitations must be acknowledged, mainly the exploratory nature and the relatively low sample size despite combining two groups from two different studies.

Once more, as in the IMvigor 211 trial, PD-L1 expression does not seem to discriminate patients who might benefit from atezolizumab. Looking for biomarkers must be mandatory to identify patients with advanced urothelial cancer who might benefit from this immune checkpoint inhibitor.

In summary, this pooled analysis of the Spanish population included in the IMvigor 210 cohort 2 and 211 trials corroborates the major hallmarks of immunotherapy. Despite not identifying statistically significant differences in ORR and OS and a worse PFS when compared with chemotherapy, atezolizumab demonstrated an unprecedented long-term benefit in those patients who responded to immunotherapy with a very favourable safety profile. Identifying potential predictive factors of response remains a critical unmet need. 
Table 3 Treatment-related adverse events
Atezolizumab

$(n=74)$

\begin{tabular}{|c|c|c|}
\hline \multicolumn{3}{|c|}{ Most common treatment-related adverse events of any grade } \\
\hline All & $44(59 \%)$ & $46(81 \%)$ \\
\hline Asthenia & $22(30 \%)$ & $28(49 \%)$ \\
\hline Pruritus & $10(14 \%)$ & $1(2 \%)$ \\
\hline Diarrhoea & $9(12 \%)$ & $8(14 \%)$ \\
\hline Decreased appetite & $7(10 \%)$ & $7(12 \%)$ \\
\hline Rash & $6(8 \%)$ & $2(3 \%)$ \\
\hline Arthralgia & $4(5 \%)$ & $5(9 \%)$ \\
\hline Decreased neutrophil count & 0 & $22(39 \%)$ \\
\hline Constipation & $2(3 \%)$ & $21(37 \%)$ \\
\hline Alopecia & 0 & $12(21 \%)$ \\
\hline Anemia & 0 & $10(18 \%)$ \\
\hline Nausea & $3(4 \%)$ & $10(18 \%)$ \\
\hline Mucosal inflammation & $1(1 \%)$ & $8(14 \%)$ \\
\hline Vomiting & $2(3 \%)$ & $7(12 \%)$ \\
\hline Abdominal pain & 0 & $7(12 \%)$ \\
\hline Nail dystrophy & $1(1 \%)$ & $5(9 \%)$ \\
\hline Decreased platelet count & 0 & $5(9 \%)$ \\
\hline Dizziness & 0 & $4(7 \%)$ \\
\hline Febrile neutropenia & 0 & $3(5 \%)$ \\
\hline Increased lacrimation & 0 & $3(5 \%)$ \\
\hline Myalgia & 0 & $3(5 \%)$ \\
\hline Paraesthesia & 0 & $3(5 \%)$ \\
\hline Pyrexia & $1(1 \%)$ & $3(5 \%)$ \\
\hline \multicolumn{3}{|c|}{ Grade 3 or 4 treatment-related adverse events } \\
\hline All & $6(8 \%)$ & $28(49 \%)$ \\
\hline Asthenia & $3(4 \%)$ & $7(12 \%)$ \\
\hline Ulcerative colitis & $1(1 \%)$ & 0 \\
\hline Haematuria & $1(1 \%)$ & 0 \\
\hline Intestinal obstruction & $2(3 \%)$ & $2(4 \%)$ \\
\hline Constipation & 0 & $5(9 \%)$ \\
\hline Decreased neutrophil count & 0 & $16(28 \%)$ \\
\hline Anaemia & 0 & $4(7 \%)$ \\
\hline Diaorrhea & 0 & $1(2 \%)$ \\
\hline Abdominal pain & 0 & $3(5 \%)$ \\
\hline Febrile neutropenia & 0 & $3(5 \%)$ \\
\hline Peripheral neuropathy & 0 & $1(2 \%)$ \\
\hline Phlebitis & 0 & $1(2 \%)$ \\
\hline Sepsis & 0 & $1(2 \%)$ \\
\hline Stomatitis & 0 & $1(2 \%)$ \\
\hline Intestinal subocclusion & 0 & $1(2 \%)$ \\
\hline General physical health deterioration & 0 & $1(2 \%)$ \\
\hline
\end{tabular}

Adverse events of all grades reported in at least $5 \%$ of patients in either group. All grade 3 or 4 are listed in both groups
Chemotherapy

$(n=57)$

$46(81 \%)$

$28(49 \%)$

$(14 \%)$

$5(9 \%)$

$22(39 \%)$

$21(37 \%)$

$12(21 \%)$

$10(18 \%)$

$10(18 \%)$

$8(14 \%)$

$(12 \%)$

$5(9 \%)$

$3 \%$

$28(49 \%)$

$16(28 \%)$

$(7 \%)$

$3(5 \%)$

$(5 \%)$

$(2 \%)$

$(2 \%)$

$1(2 \%)$ 
Acknowledgements The authors acknowledge the contribution of all patients, centers and PIs participating in this study: Dr. CastellanoHospital Universitario 12 de Octubre, Dr. Pérez Gracia-Clínica Universitaria de Navarra, Dr. Alonso and Dr. Gajate-Hospital Ramón y Cajal, Dr. Pérez Valderrama and Dr. Durán Martínez-Hospital Universitario Virgen del Rocío, Dr. García del Muro-Institut Catala d Oncologia Hospital Duran i Reynals, Dr. Arranz-Hospital General Universitario Gregorio Marañón, Dr. Morales-Hospital Universitari Vall d'Hebron, Dr. Gallardo-Corporacio Sanitaria Parc Tauli, Dr. Puente-Hospital Universitario Clínico San Carlos, Dr. Mellado-Hospital Clinic i Provincial, Dr. Mellado-Hospital de la Santa Creu i Sant Pau, Dr. Lainez-Hospital de Navarra, Dr. Mendez-Hospital Universitario Reina Sofia, Dr. Montesa-Hospital Clínico Universitario Virgen de la Victoria, Dr. Caballero-Hospital General Universitario de Valencia, Dr. García-Complejo Hospitalario Universitario de Santiago (CHUS), Dr. González Del Alba-Hospital Universitario Son Espases, Dr. Borrega-Hospital San Pedro de Alcántara, Dr. Chirivella-Hospital Clínico Universitario de Valencia. The authors are grateful to Syntax for Science, S.L. for their technical assistance in the analysis, writing and submission of this manuscript.

Author contributions Introduction and discussion M.S. and I.D. All authors have participated in this analysis, but especially in revising it critically and all authors have approved the final article.

Funding This study was funded by Roche Farma, Spain.

\section{Compliance with ethical standards}

Conflict of interest Teresa Alonso Gordoa has received honoraria for consultancy/advisory or speaker from Ipsen, Pfizer, Roche/Genentech, Sanofi, Bayer, Astellas, Janssen-Cilag, Merck Sharp \& Dohme, Bristol-Myers Squibb and Eisai. Pablo Gajate has received honoraria for participation in advisory boards from Roche; as a speaker from Pfizer, Bristol-Myers Squibb and Roche/Genentech; and travel expenses from Pfizer and Roche/Genentech. Enrique Gallardo has received honoraria for Consultant or Advisory Role: Sanofi, Janssen, Astellas, Pfizer, Bayer, Roche, Ipsen, Novartis, Eisai, EUSA Pharma, BMS, AstraZeneca, Merck, Rovi, Daiichi Sankyo, Techdow. Speaking Role: Astellas, Janssen, Sanofi, Bayer, Ipsen, Pfizer, Roche, BMS,Novartis, Rovi, Daiichi Sankyo, Leo Pharma, Menarini, Eisai, MSD, Boehringer, Ingelheim, Merck. Grant support (personal/institutional): Astellas, Janssen, Sanofi, Pfizer, Bayer,Ipsen, Roche, Ferrer, GSK, BMS. Travel expenses: Astellas, Janssen, Sanofi, Bayer, Ipsen, Roche, Novartis, Pierre Fabre, Pfizer, Eisai. Rafael Morales Barrera has received honoraria for consulting, advisory and/or speaker bureaus from Sanofi, Bayer, Janssen, AstraZeneca, Merck Sharp \& Dohme, Asofarma; and received travel and accommodations expenses from Roche/Genentech, Sanofi, Astellas, Janssen, Merck Sharp \& Dohme, Bayer, Pharmacyclics, Clovis and Eli Lilly. Jose Luis Pérez Gracia has received research grants and support from Roche, Bristol-Myers Squibb, Merck Sharp \& Dohme, Ipsen, Eisai, Incyte and Janssen; honoraria as a speaker and as part of advisory boards from Roche/Genentech, Bristol-Myers Squibb, Ipsen, Eisai, Merck Sharp \& Dohme and Seattle Genetics; and travel support from Roche/Genentech, Merck Sharp \& Dohme and Bristol-Myers Squibb. Javier Puente has received honoraria as consultant/advisor or speaker from Pfizer, Astellas, Janssen, MSD, Bayer, Roche/Genentech, Bristol-Myers Squibb, Boehringer, AstraZeneca, Ipsen, Novartis, Eusa Pharma, Eisai, Pierre-Fabre, Merck and Sanofi; and research funding from Astellas, Pfizer and Roche/Genentech. Ignacio Durán has received honoraria from Bristol-Myers Squibb, Ipsen, Roche/Genentech, Janssen, MSD, Astellas and Eusa Pharma; consulting/advisory boards from Roche/Genentech, Merck Sharp \& Dohme, Bayer, Bristol-Myers
Squibb, Seattle Genetics, Pharmacyclics, Janssen and Novartis; research funding from Roche/Genentech, AstraZeneca.

Ethical approval All procedures performed in studies involving human participants were in accordance with the ethical standards of the institutional reserach committee and with the 1964 Helsinki declarartion and its later ammendments or comparable ethical standards.

Informed consent Informed consent was obtained from all individual participants included in the study.

Consent to participate Patients already gave their consent to participate in the corresponding trials.

Consent for publication All authors have consented to submission of this article for publication.

Availability of data and material "Qualified researchers may request access to individual patient level data through the clinical study data request platform (https://vivli.org/). Further details on Roche's criteria for eligible studies are available here. (https://vivli.org/members/ourme mbers/). For further details on Roche's Global Policy on the Sharing of Clinical Information and how to request access to related clinical study documents, see here. (https://www.roche.com/research_and_devel opment/who_we_are_how_we_work/clinical_trials/our_commitment _to_data_sharing.htm $). "$

Open Access This article is licensed under a Creative Commons Attribution 4.0 International License, which permits use, sharing, adaptation, distribution and reproduction in any medium or format, as long as you give appropriate credit to the original author(s) and the source, provide a link to the Creative Commons licence, and indicate if changes were made. The images or other third party material in this article are included in the article's Creative Commons licence, unless indicated otherwise in a credit line to the material. If material is not included in the article's Creative Commons licence and your intended use is not permitted by statutory regulation or exceeds the permitted use, you will need to obtain permission directly from the copyright holder. To view a copy of this licence, visit http://creativecommons.org/licenses/by/4.0/.

\section{References}

1. Observatory Global Cancer. (Online). 2018 (cited 2020 Enero). Available from: https://globocan.iarc.fr/.

2. WHO. A. Cancer incidence in five continents. (Online). 2019 (cited 2019 January 19). Available from: https://ci5.iarc.fr/ Default.aspx.

3. Bellmunt J, Orsola A, Leow JJ, et al. Bladder cancer: ESMO practice guidelines for diagnosis, treatment and follow-up. Ann Oncol. 2014;25(Suppl 3):iii40-iii48.

4. Flaig TW, Spiess PE, Agarwal N, et al. Bladder Cancer, Version 3.2020, NCCN Clinical Practice Guidelines in Oncology. J Natl Compr Canc Netw. 2020;18(3):329-54.

5. von der Maase H, Hansen SW, Roberts JT, et al. Gemcitabine and cisplatin versus methotrexate, vinblastine, doxorubicin, and cisplatin in advanced or metastatic bladder cancer: results of a large, randomized, multinational, multicenter, phase III study. J Clin Oncol. 2000;18(17):3068-77.

6. Galsky MD, Hahn NM, Rosenberg J, et al. Treatment of patients with metastatic urothelial cancer "unfit" for cisplatin-based chemotherapy. J Clin Oncol. 2011;29(17):2432-8. 
7. De Santis M, Bellmunt J, Mead G, et al. Randomized phase II/ III trial assessing gemcitabine/carboplatin and methotrexate/carboplatin/vinblastine in patients with advanced urothelial cancer who are unfit for cisplatin-based chemotherapy: EORTC study 30986. J Clin Oncol. 2012;30(2):191-9.

8. Gómez De Liaño A, Duran I. The continuing role of chemotherapy in the management of advanced urothelial cancer. Ther Adv Urol. 2018;10(12):455-80.

9. Berdik C. Unlocking bladder cancer. Nature. 2017;551(7679):S34-S3535.

10. Sankin A, Narasimhulu D, John P, et al. The expanding repertoire of targets for immune checkpoint inhibition in bladder cancer: what lies beneath the tip of the iceberg, PD-L1. Urol Oncol. 2018;36(10):459-68.

11. Chen DS, Irving BA, Hodi FS. Molecular pathways: nextgeneration immunotherapy-inhibiting programmed death-ligand 1 and programmed death-1. Clin Cancer Res. 2012;18(24):6580-7.

12. Dong H, Strome SE, Salomao DR, et al. Tumor-associated B7-H1 promotes T-cell apoptosis: a potential mechanism of immune evasion. Nat Med. 2002;8(8):793-800.

13. Herbst RS, Soria JC, Kowanetz M, et al. Predictive correlates of response to the anti-PD-L1 antibody MPDL3280A in cancer patients. Nature. 2014;515(7528):563-7.

14. Powles T, Eder JP, Fine GD, et al. MPDL3280A (anti-PD-L1) treatment leads to clinical activity in metastatic bladder cancer. Nature. 2014;515(7528):558-62.

15. Rosenberg JE, Hoffman-Censits J, Powles T, et al. Atezolizumab in patients with locally advanced and metastatic urothelial carcinoma who have progressed following treatment with platinumbased chemotherapy: a single-arm, multicentre, phase 2 trial. Lancet. 2016;387(10031):1909-20.

16. Powles T, Durán I, van der Heijden MS, et al. Atezolizumab versus chemotherapy in patients with platinum-treated locally advanced or metastatic urothelial carcinoma (IMvigor211): a multicentre, open-label, phase 3 randomised controlled trial. Lancet. 2018;391(10122):748-57.

17. Bellmunt J, Fougeray R, Rosenberg JE, et al. Long-term survival results of a randomized phase III trial of vinflunine plus best supportive care versus best supportive care alone in advanced urothelial carcinoma patients after failure of platinum-based chemotherapy. Ann Oncol. 2013;24(6):1466-72.
18. Bellmunt J, Théodore C, Demkov T, et al. Phase III trial of vinflunine plus best supportive care compared with best supportive care alone after a platinum-containing regimen in patients with advanced transitional cell carcinoma of the urothelial tract. J Clin Oncol. 2009;27(27):4454-61.

19. González Del Alba A, De Velasco G, Lainez N, et al. SEOM clinical guideline for treatment of muscle-invasive and metastatic urothelial bladder cancer (2018). Clin Transl Oncol. 2019;21(1):64-74.

20. Balar AV, Galsky MD, Rosenberg JE, et al. Atezolizumab as first-line treatment in cisplatin-ineligible patients with locally advanced and metastatic urothelial carcinoma: a single-arm, multicentre, phase 2 trial. Lancet. 2017;389(10064):67-766.

21. Bellmunt J, de Wit R, Vaughn DJ, et al. Pembrolizumab as secondline therapy for advanced urothelial carcinoma. N Engl J Med. 2017;376(11):1015-26.

22. Khaki AR, Li A, Diamantopoulos LN, et al. Impact of performance status on treatment outcomes: a real-world study of advanced urothelial cancer treated with immune checkpoint inhibitors. Cancer. 2020;126(6):1208-16.

23. Sternberg CN, Loriot Y, James N, et al. Primary results from SAUL, a multinational single-arm safety study of atezolizumab therapy for locally advanced or metastatic urothelial or nonurothelial carcinoma of the urinary tract. Eur Urol. 2019;76(1):73-81.

24. Gyawali B, Hey SP, Kesselheim AS. A Comparison of Response patterns for progression-free survival and overall survival following treatment for cancer with PD-1 inhibitors: a meta-analysis of correlation and differences in effect sizes. JAMA Netw Open. 2018;1(2):e180416.

25. Sonpavde G, Pond GR, Fougeray R, et al. Time from prior chemotherapy enhances prognostic risk grouping in the second-line setting of advanced urothelial carcinoma: a retrospective analysis of pooled, prospective phase 2 trials. Eur Urol. 2013;63(4):717-23.

26. Motzer RJ, Rini BI, McDermott DF, et al. Nivolumab plus ipilimumab versus sunitinib in first-line treatment for advanced renal cell carcinoma: extended follow-up of efficacy and safety results from a randomised, controlled, phase 3 trial. Lancet Oncol. 2019;20(10):1370-85.

Publisher's Note Springer Nature remains neutral with regard to jurisdictional claims in published maps and institutional affiliations. 